\title{
Analysis of High-Frequency QRS Components Obtained from Body Surface Potential Mapping in Anterior Myocardial Infarction
}

\author{
MS Guillem ${ }^{1}$, J Millet $^{1}$, V Bodí ${ }^{2}$, A Bollmann $^{3}$ \\ ${ }^{1}$ UPV, Universidad Politécnica de Valencia, Valencia, Spain \\ ${ }^{2}$ HCUV, Hospital Clínico Universitario de Valencia, Valencia, Spain \\ ${ }^{3}$ Department of Cardiology, Otto-von-Guericke-University, Magdeburg, Germany
}

\begin{abstract}
Analysis of high frequency components of the QRS complex in the standard twelve-lead ECG has been suggested as indicator for ischemia, as significant changes in morphology and voltage amplitude have been observed. Changes in high frequency components for old myocardial infarction are less specific and their advantages over the unfiltered ECG are unclear. However, lead positions outside the standard ECG have not been explored systematically. In this study, we extend the number of leads included in high frequency $Q R S$ analysis looking for differences between controls and old anterior myocardial infarctions by applying body surface mapping. Differences in the number of leads presenting reduction in the high frequency content of the QRS complex, as well as the temporal width of this reduction. have been found between patients and normals, suggesting markers for electrocardiographic assessment of conduction slowing.
\end{abstract}

\section{Introduction}

During the last years, significant progress has been made in the study of high frequency components of the QRS complex of normal subjects and patients with myocardial pathologies.

It is widely accepted that during coronary occlusion some changes are visible in high frequency components of the QRS complex. RMS voltage values may decrease significantly and some notches and slurs appear in the envelope of high frequency signals [1]. In consequence, all changes found are related to a decrease in the high frequency content of the QRS complex. This finding has been associated with a reduction in conduction velocity [2]

Controversial results have been observed when studying high-frequency components of the QRS complex in patients with an old myocardial infarction. Theoretically, high frequency content of the QRS complex may decrease, due to conduction velocity reduction derived from peri-infarction block. While some authors have observed this [3-4], others have not found differences between patients with infarction and normal subjects [5] or even have reported some increase in the high-frequency content [6].

However, lead positions outside the standard ECG have not been explored systematically yet. A more detailed description of high-frequency components of the QRS complex in the whole body surface could elucidate differences between controls and post-infarction patients with independence of the leads explored and of the specific injured area in the heart.

In this study, we extend the number of leads included in high frequency QRS analysis for comparing healthy subjects and post-infarction patients using parameters previously defined for limited lead sets: voltage RMS values and presence or absence of temporary voltage reductions. Additionally, after having observed different morphologies of high frequency QRS components of both controls and post-infarction patients, we define additional parameters for distinguishing both groups.

\section{Patients and methods}

\subsection{Study population}

We prospectively studied 23 subjects, 7 of whom had no cardiac pathologies (control group) and 16 patients with a first $\mathrm{Q}$ wave anterior myocardial infarction. All patients and controls gave informed consent. The normal group consisted of 7 men with no evidence of heart disease by history, physical examination and 12-lead electrocardiogram.

All patients were also male admitted in the HCUV because of a first Q wave myocardial infarction. Myocardial infarction was diagnosed on the basis of typical chest pain lasting $>30$ minutes, ST elevation $>0.1$ $\mathrm{mV}$ presented in at least two leads from the 12-lead ECG, and an increase in troponin I $>1 \mathrm{ng} / \mathrm{ml}$. Patients were eligible for inclusion if they fulfilled the following inclusion criteria: 1) Stable clinical course without complications. 2) Q waves of $\square 0.04$ s duration and $\square 25 \%$ of the amplitude of the $\mathrm{R}$ wave in depth in two or more 
contiguous leads. 3) Absence of any condition known to provoke ST segment alterations. 4) Absence of clinical history suggesting any prior heart disease. 5) Singlevessel disease and a patent (with a residual lesion <50\%) infarct-related artery at the end of the cardiac catheterization which was performed routinely before hospital discharge.

\subsection{Body surface potential mapping system description}

Body surface potential mapping (BSPM) was performed 6 months after the index myocardial infarction. The system used to record electrocardiographic signals has been previously described [7]. Briefly, it makes use of a commercial 64-lead acquisition system for biopotential measurements (Active One, Biosemi, Amsterdam, Netherlands) adapted to our purposes. In this system, all acquired potentials are referred to one electrode defined as the electrical reference. Electrocardiographic signals are sampled at $2048 \mathrm{~Hz}$ with an amplitude resolution of $1 \square \mathrm{V} /$ bit. BSPM recordings lasting 60 second were stored on hard disk for off-line processing. The bandwidth of the system is $512 \mathrm{~Hz}$ (is it correct to relate to "bandwith" with only one parameter?), which is enough for highfrequency QRS analysis.

Electrodes are distributed non-uniformly upon the chest: a set of 16 electrodes is placed uniformly in the back and the rest non-uniformly in the anterior side, with the highest density in positions overlaying the heart. Electrode positions are depicted in Figure 1. Electrodes are mounted on an elastic vest designed by our group for fast and easy attachement to the patient's body.

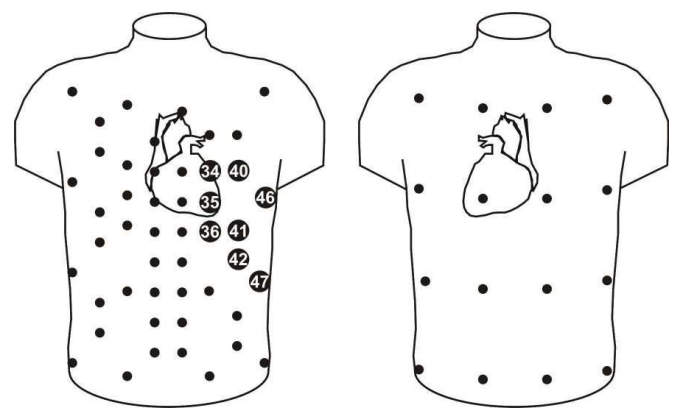

Figure 1: Electrode configuration. Position of the electrodes are marked by dots. Left panel: chest. Right panel:back. Numbered electrodes belong to the left anterior zone.

\subsection{Signal processing}

By means of specific applications developed under MATLAB 6.5 (The Mathworks, Natik, USA), signals were conditioned and analyzed.
First, the electrical reference, which was fixed in one electrode, is changed. Wilson Central Terminal lead is computed by using chest leads closer to limbs and then this reference is subtracted from all chest leads.

Baseline fluctuations are removed using a linear high pass filter $(\mathrm{fc}=1 \mathrm{~Hz})$. Power spectrum density is then computed for each lead using the Welch's periodogram, with 32768 points and a hamming window of 16384 points, with an overlap of $50 \%$. Leads presenting $50 \mathrm{~Hz}$ components greater than $0.5 \%$ are filtered using a notch filter with $1 \mathrm{~Hz}$ bandwidth.

Then, a first averaged beat (PQRST) is computed for each lead and QRS onset and offset are detected for each lead. These fiducial points are used to define the window for creating QRS averaged complexes, computed with a template matching algorithm. All beats are grouped according to their similarity, using a cross-correlation function with a coefficient threshold of 0.97. A template is generated for each lead by averaging all the beats belonging to the most numerous group. QRS templates are accepted only if generated with more than 25 beats.

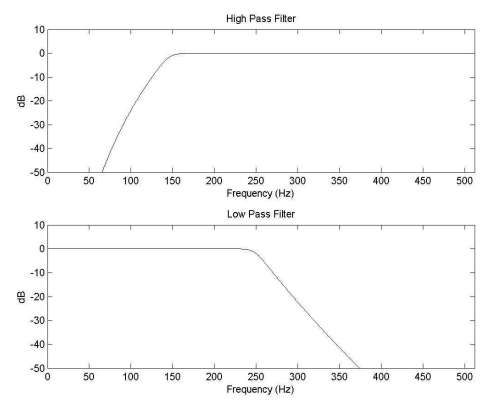

Figure 2: Filters used

QRS complexes are then filtered in order to emphasize frequencies between 150 and $250 \mathrm{~Hz}$ by using two butterworth filters, whose frequency responses are shown in Figure 2.

The envelope of filtered QRS complexes is computed in order to obtain specific measurements. For that purpose, rectified signals are low-pass filtered, with a butterworth filter $(\mathrm{fs}=132 \mathrm{~Hz})$.

\subsection{HF-QRS measurements}

For each filtered QRS complex RMS voltage value is computed as follows:

$$
\mathrm{RMS}=\sqrt{\frac{\sum_{\mathrm{k}=\mathrm{QRS}_{\text {on }}}^{\mathrm{QRS}_{\text {off }}} \mathrm{QRSf}_{\mathrm{k}}^{2}}{\mathrm{RS}_{\text {off }}-\mathrm{QRS}_{\text {on }}}}
$$

Then, the mean value for each lead is computed for the two groups: patients and controls and the voltage reduction for the infarction group is computed as the difference between mean values for control and 
patientsfor each lead. Also, the mean voltage RMS reduction was computed for the left anterior electrodes (Figure 1), as this zone has shown greater differences between patient and normal groups.

Using the envelope of filtered QRS, the presence of "wide" reduced amplitude zones (RAZs) was assessed.

Typically, a RAZ is defined to be present when two or more local maxima of the envelope are found. This finding may be indicative of ischemia, and should subsequently not be present in healthy subjects. A typical filtered QRS complex for a healthy subject can be observed in Figure 3. Only one maximum of the positive/negative envelope appears. The definition of local maxima is each envelope point whose amplitude value exceeds the amplitude of the three envelope points immediately preceding and following it. Also, as defined by the NASA, a RAZ needs to meet an additional criterion: the amplitude of the secondary local maximum has to be of at least $30 \%$ of the amplitude of the primary maximum [8].

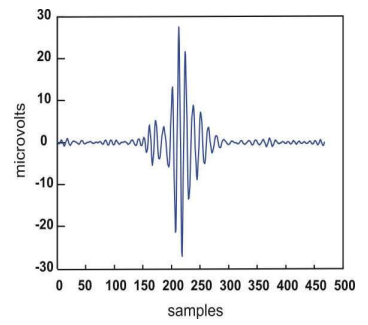

Figure 3: Typical HF QRS of a normal subject acquired in the left anterior area

\section{Novel measurements}

We have introduced a new measurement: RAZ width. We define the width of a RAZ at half height, as presented in Figure 4, taking the height as the amplitude difference between the second envelope maximum and the minimum envelope value in the RAZ. (See Figure 4). Additionally, we define a "wide RAZ" as any RAZ presenting a width greater than $10 \mathrm{~ms}$.

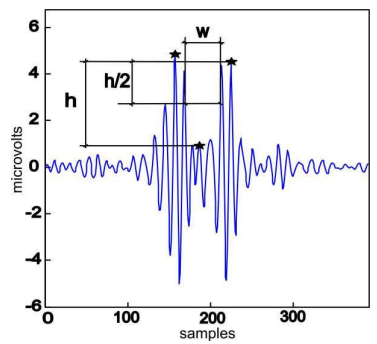

Figure 4: Definition of RAZ width

Results for each patient are grouped according to the location of the lead. We divide them into left anterior or non-left anterior, according to lead positions shown in Figure 1.

\section{Results}

Globally, patients with infarction showed a RMS voltage reduction of $1.0 \pm 0.9 \mu \mathrm{V}$ in the left anterior area. Voltage reduction for each lead is presented in Table 1.

Table 1: RMS voltage (VRMS) values for left anterior leads in controls and patients

\begin{tabular}{ccc}
\hline Lead & Control & Patients \\
\hline 34 & $4.6 \pm 1.5 \mu \mathrm{V}$ & $4.8 \pm 1.6 \mu \mathrm{V}$ \\
35 & $5.9 \pm 1.5 \mu \mathrm{V}$ & $4.6 \pm 2.2 \mu \mathrm{V}$ \\
36 & $5.7 \pm 2.1 \mu \mathrm{V}$ & $4.1 \pm 2.0 \mu \mathrm{V}$ \\
40 & $5.0 \pm 1.5 \mu \mathrm{V}$ & $4.6 \pm 1.4 \mu \mathrm{V}$ \\
41 & $6.7 \pm 2.2 \mu \mathrm{V}$ & $4.1 \pm 1.5 \mu \mathrm{V}$ \\
42 & $5.1 \pm 2.4 \mu \mathrm{V}$ & $4.0 \pm 1.4 \mu \mathrm{V}$ \\
46 & $5.8 \pm 2.6 \mu \mathrm{V}$ & $4.0 \pm 1.2 \mu \mathrm{V}$ \\
47 & $3.7 \pm 1.7 \mu \mathrm{V}$ & $3.7 \pm 1.5 \mu \mathrm{V}$ \\
\hline
\end{tabular}

All patients and controls exhibited a number of entire body surface leads with RAZs (16.1 \pm 9.33 in controls vs $15.2 \pm 11.5$ in patients, $\mathrm{p}=\mathrm{ns}$ ). Considering only the left anterior zone, the number of controls with RAZs was 3 (43\%) while the number of patients with RAZs was 12 (75\%). The number of leads with RAZs was $2.7 \pm 0.6$ for the control group versus $2.3 \pm 1.2$ for the patient group $(\mathrm{p}=\mathrm{ns})$.

Table 2: RAZs in controls and patients with infraction in the total body surface vs left anterior area

\begin{tabular}{ccccc}
\hline & \multicolumn{2}{c}{ Subjects with RAZ } & \multicolumn{2}{c}{ Leads with RAZ } \\
& Total & Anterior & Total & Anterior \\
\hline $\begin{array}{c}\text { Controls } \\
n=7\end{array}$ & $7(100 \%)$ & $3(43 \%)$ & $16.1 \pm 9.33$ & $2.7 \pm 0.6$ \\
$\begin{array}{c}\text { Patients } \\
n=16\end{array}$ & $16(100 \%)$ & $12(75 \%)$ & $15.2 \pm 11.5$ & $2.3 \pm 1.2$ \\
\hline
\end{tabular}

Table 3 shows the presence of wide RAZs (wRAZ) in patients and controls. $43 \%$ of control subjects presented at least one lead with a wRAZ, while $63 \%$ of patients had at least one lead with a wRAZ. All control subjects with wRAZs, presented them in one lead per patient, with a mean duration of $11.2 \pm 0.6 \mathrm{~ms}$. Patients showed wRAZs in more leads $(2.7 \pm 1.8)$ with a slightly longer duration (13.5 $\pm 2.4 \mathrm{~ms})$.

Table 3: Wide RAZs in left anterior leads.

\begin{tabular}{cccc}
\hline & $\begin{array}{c}\text { Subjects } \\
\text { with wRAZ }\end{array}$ & $\begin{array}{c}\text { Leads with } \\
\text { wRAZ }\end{array}$ & $\begin{array}{c}\text { wRAZ } \\
\text { width (ms) }\end{array}$ \\
\hline $\begin{array}{c}\text { Controls } \\
\mathrm{n}=7\end{array}$ & $3(43 \%)$ & $1 \pm 0$ & $11.2 \pm 0.6$ \\
$\begin{array}{c}\text { Patients } \\
\mathrm{n}=16\end{array}$ & $10(63 \%)$ & $2.7 \pm 1.8$ & $13.5 \pm 2.4$ \\
\hline
\end{tabular}




\section{Discussion}

This study explored differences of high frequency components obtained from BSMP between patients with previous anterior myocardial infarction and normal controls

RMS voltage reduction has been found in some left anterior leads when comparing controls and patients. The high frequency content seems to decrease noticeably in some leads of the left precordial area: e.g. lead 35, 36, 41 and 46. However, significance of this voltage reduction is limited due to the great differences among subjects of each group, especially for the control group. Although HF-QRS analysis has been found to be sufficiently reproducible for clinical use (Batdorf $\mathrm{NJ}$ et al. J Electrocardiol. 2004 Oct;37(4):289-96.), the interindividual variation of HF-QRS has, in accordance with our findings, been shown to be large in normals and patient groups. (Tragardh E et al. J Electrocardiol. 2004 Jul;37(3):157-62)

The number of leads presenting RAZs in the total body surface with the NASA criterion has not been proven to be useful, as every control subject showed a number of leads with temporal high frequency content reduction. However, we have noticed that differences can be observed when studying the left anterior area separately. Only 43\% control subjects presented RAZs in left anterior leads, while $75 \%$ AMI patients presented this sign.

Although the number of leads presenting RAZs in patients and controls was comparable, differences were found in the high frequency content of both groups of patients expressed as RAZ width: most RAZs of patients with myocardial infraction were deeper and wider. The number of left anterior leads presenting wide RAZs in the control group was lower than in the infarction group and the width of these was also smaller). While this finding is novel, by studying established parameters Ringborn et al. [8] observed no significant differences between normals and patients with previous infarction in the summed HFQRS of all 12 leads or in the pattern of lead distribution of the HF-QRS.

\section{Study limitations}

A satisfactory performance of the averaging algorithm, necessary to obtain the true high frequency components of electrocardiographic signals, require long low-noise recordings. This requirement made us discard most of our previous, shorter recordings, and the number of patients included in this analysis was lower than what we expected, specially for the control group.

Analysis of a higher number of patients, with various infarction locations and longer recordings are necessary to prove these preliminary results observed in a small number of subjects.

\section{Conclusion}

Reduction of high frequency content of the QRS complex has been found in patients with an old anterior myocardial infarction. Although small differences have been found in the entire body surface between patient and control groups, most prominent changes have been observed in the left anterior area of the torso. Lower RMS voltage values, higher incidence of leads presenting with RAZs and the novel finding of higher number of leads with wide RAZs are present in myocardial infarction patients. Since RAZ are suggested to be related with slowing of conduction velocity (Watanabe $\mathrm{T}$ et al. Jpn Circ J. 1998 Nov;62(11):844-8.)in peri-myocardial areas further analysis is warranted as these findings could prove to be useful markers for electrocardiographic assessment of arrhythmic risk.

\section{Acknowledgements}

This work was supported by TIC2002-009 project. Authors wish to thank the HCUV staff.

\section{References}

[1] Mor-Avi V, Shargorodsky B, Abboud S et al. Effects of coronary occlusion on high-frequency components of the epicardial electrogram and body surface electrocardiogram. Circulation 1987; 76:237-43.

[2] Pettersson J, Pahlm O, Carro E, et al. Changes in highfrequency QRS components are more sensitive than STsegment deviation for detecting acute coronay artery occlusion. J Am Coll Cardiol 2000; 36(6):1827-34.

[3] Anderson GJ, Blieden MF. The high-frequency electocardiogram in coronary artery disease. Am Heart J 1975;89:349-58.

[4] Goldberger AL, Bhargava V, Froelicher et al. Effect of myocardial infarction on high-frequency QRS potentials. Circulation 1981;64:34-42.

[5] Ringborn M, Pahlm O, Wagner GS et al. The absence of high-frequency QRS changes in the standard electrocardiographic QRS changes of old myocardial infarction. Am Heart J. 2001;141:573-9.

[6] Novak P, Novak V et al. Time-frequency mapping of the QRS complex in normal subjects and in postmyocardial infarction patients. J Electrocardiol 1994;27:49-60.

[7] Guillem, M. S., Millet, J., Bodí, V., Mora, C., and Chorro, F. J. Integration of a Body Surface Potential Mapping System. X Mediterranean Conference on Medical and Biological Engineering. 2004. [in press]

[8] Schlegel TT, Kulecz WB, DePalma JL et al. Real Time 12lead high-frequency QRS electrocardiography for enhanced detection of myocardial ischemia and coronary artery disease. Mayo Clin Proc 2004;79:339-50.

Address for correspondence.

María de la Salud Guillem Sánchez

mguisan@doctor.upv.es 